\section{A \\ on}

\section{THE DIAGNOSIS AND TREATMENT OF SPINAL CORD TUUIOURS."}

\author{
BY
}

DONALD J. ARMOUR, C.M.G., F.R.C.S.,

Senior Surgeon, West London Hospital; Surgeon, National Hospital for Nervous Diseases.

Is this discussion on the diagnosis and treatment of spinal cord tumours I will limit myself to the strict meaning of the term-namely, those tumours arising from the cord, from the nerve roots, or from the spinal meninges-and exclude those arising, primarily or secondarily, from the vertebral column and the soft tissues surrounding it. Exception will be made in the case of chondromata growing from an intervertebral disc.

Clinically, spinal cord tumours may be said to cause a paraplegia by compression, the nature of which cannot be revealed by $x$ rays. It should be remembered, however, that tumours arising from nerves or nerve roots outside the spinal canal may extend into it through an intervertebral foramen, and, vice versa, one originating in the spinal canal may extend in the same way into the soft tissues surrounding the vertebial column. Moreover, a tumour may be both intradural and extradural, without any clear evidence as to its point of origin. More than three-fourths of spinal cord tumours are extramedullary, and less than one-fourth intramedullary. Of the extramedullary tumours, the intradural are more frequent than the extradural by three to one. Of intradural tumours, about two-thirds occupy a dorsal or dorso-lateral position and one-third a ventral or ventro-lateral position-a matter for rejoicing on the part of the surgeon.

\section{Sумртомs.}

In the majority of cases of spinal cord tumour the onset of symptoms is slowly progressive, the course of the disease being slow and chronic. From the clinical standpoint it may be divided into three stages: (1) of root symptoms, usually unilateral; (2) of Brown-Séquard paralysis; (3) of complete compression paraplegia, or bilateral paralysis. According to Starr, " a certain order is commonly observed in the symptoms" -namely: (1) Pain referred to the periphery. (2) Increase of reflex activity below the point of compression. (3) Paralysis-spastic paraplegia in extension. (4) Loss of sensibility. (5) Loss of reflex activity with flaccid paralysis.

The first symptom complained of by the patient is usually scusory, either pain or some form of paraesthesia. There are exceptions to this, but though at the onset some cases have no sensory symptoms, they usually appear later in the course of the disease. A few cases run a completely painless course. These were formerly supposed to be cases of medullary tumour, but it is now known that some are extranedullary.

Root pain, due to irritation- "the neuralgic stage"is the most frequent early symptom. "There is no other disease which causes such definitely localized recurring and persistent pain" (Starr). - It may be paroxysmal and remittent, showing marked variations in its severity, and is frequently aggravated by coughing and sncezing or by movements of the spine. The pain may appear suddenly, may persist for months or years, disappearing as symptoms of cord compression appear, or it may continue throughout the whole duration of the disease. The pain is referred to the course of the nerve involved, and nay be thought to be due to innumerable conditions in various parts of the bodr or limbs.

Pain in the back may be an early symptom in spinal cord tumour, and may be the only one complained of for a very long period. This is especially true of lunibo-sacial and cauda equina tumours. Various postures may be assumed by the patient to get relief from the pain.

Bennett ${ }^{i}$ has called attention to the fact that orthopaedic surgeons are frequently consulted by patients com-

* Read in opening a discussion in the Section of Surgery of the Annual Meeting of the British Medical Association, Cardiff, 1928. plaining of local and referred pains in the region of the spine who have no bone lession but a lesion of the cord. He records four cases of spinal cord tumour, all having shown : symptoms that suggested osseous, muscular, and ligamentous changes rather than any neurological condition. They showed (1) variable pain in the spine and nerve roots; (2) extreme spasm of the erector spinae muscles, followed by lateral deformity of the spine; (3) extreme bilateral spasm of the hamstring group-all appearing long before any motor or sensory changes had occurred.

Pain, not radicular in character or distribution, may be complained of in a part of the body not supplied by the roots involved in the tumour. This pain is due to pressure on the sensory tracts of the cord. It may antedate any discoverable sensory or motor changes.

Paraesthesiae of . various kinds--" pins and needles," numbness, a feeling of weight, tingling, cold-may be complained of. They may occur at the level of the tumour, due to root irritation; or above the level of the tumour, due to pressure from the held-up cerebro-spinal fluid or to sympathetic involvement (Barré and Schrapf); or below the tumour level, due to irritation of sensory tracts in the cord. Elsberg says that a complaint of a feeling of coldness in the extremities is a regular symptom of high cervical tumours.

In the early stages of spinal tumour objective sensory changes are usually absent, though areas of hyperaesthesia may be found on careful search. Later, the cutaneous sensory disturbances will depend upon the amount of compression or destruction of the spinal tracts, and will vary with the site of the tumour and its relation to the cord. Elsberg calls attention to the frequent occurrence in extramedullary tumours of a certain amount of dissociation of sensations below the level of the tumour, while in intrameduliary tumours, on the other hand, he frequently found that all types of sensation were equally involved, while typical dissociation was rarely seen. He points out, however, that unless there is a loss of all sensation below the segmental level, there is a striking difference between the sensory disturbances in extramedullary and intramedullary tumours. It is this: that in extramedullary growths the maximum of sensory disturbance is in the most peripheral dermatomes and the minimum nearest the level of the lesion; while in intramedullary growths the maximum of sensory disturbance is often at and below the segmental level.

Unless all cutaneous sensation has been lost, tactile sensation is usually less affected than pain and temperature, which are often equally diminished. But mans variants of this occur. In tumours of the conus and cauda equina the sensory disturbances are usually bilateral and asymmetrical, affecting all types of sensation to an equal degree.

Subjective motor disturbance, consisting of a complaint of weakness followed by stiffness, usually follows subjective sensory symptoms, though very rarely the reverse occurs. These symptoms of weakness and stiffness are usually progressive, though cases are reported in which they have been remittent. In high tumours the order of limb involvement is almost always in regular sequence-namely, homolateral upper limb, homolateral lower limb, contralateral lower limb, contralateral upper limb.

During the first or irritative stage, if the tumour involves an anterior root there should be motor excitement, as shown by tremor, muscular tension, spasms or general tonic spasms. But by the time the patient comes under observation these are generally absent, or soon masked or suppressed by a degenerative paralysis of the corresponding muscles, together with a diminished tendon reflex. Obvious wasting of the muscles concerned usually follows, and this whether the root or the anterior horn cells are suffering from pressure.

At first, as the tumour lies on one side of the cord, its pressure may cause a-spinal hemiparesis, the so-called Brown-Séquard syndrome, characterized by motor disturbances on the same side as the lesion and sensory changes on the opposite side. This is rarely seen, however, in its complete form in tumour compression, and soon passes into a paraparesis or paraplegia. Elsberg has described what 
he calls a " reversed " Brown-Séquard syndrome, in which the motor and sensory disturbances are opposite to those described. The explanation of this would appear to be the pushing of the cord over against the opposite side of the canal by the growing tumour.

As soon as the tumour begins to exert further pressure on the cord two sets of spinal symptoms begin to appearnamely, (1) direct spinal symptoms, due to compression or destruction of the segments on which or in which the tumour lies; (2) indirect spinal symptoms, due to pressure upon the long tracts traversing the segment affected. Thus the atrophy and paralysis of muscles, often with fibrillary twitchings, the segmental supply being known, with reaction of degeneration and loss of reflex activity in the muscle tendons, combined with muscular weakness and rigidity and increased reflex activity below the lesion, an extersor plantar response, with anaesthesia up to the level of the lesion, and probably loss of bladder and rectum control, constitute a condition of great diagnostic value.

In these cases not only does a minimal stimulus produce the corresponding reflex, but very often a number of other reflex contractions-the so-called "mass reflexes" described by Riddoch, and called by Babinski "defence reflexes." Usually sphincter disturbances do not appear until motor and sensory symptoms are well advanced, though they may be early in onset in intramedullary tumours. Bladder loss is usually the first to appear, and precedes the rectal loss by some time. Very often " imperative micturition" appears first, or hesitancy passes into retention, and retention into incontinence.

It is interesting to remember here that it has been shown by Riddoch and Purves-Stewart that the pathological effect of the actual pressure on the cord elements are extraordinarily limited, the neural processes of inflammation, softening, and degeneration being confined to the immediate site of the tumour, and that the widespread interference with cord function is due to vascular changes, setting $u$ p what they call anoxaemia. That this must be true is shown by the recovery which follows, often rapidly, upon the removal of a tumour which has caused very great narrowing and distortion of the cord.

\section{Paraplegia in Flexion.}

This condition has been very fully worked out and described by Walshe. It follows most frequently upon paraplegia in extension, but in a certain number of cases the extension stage is very short and the paraplegia in flexion may appear to be the original condition. It is characterized by: (1) Flexion of the different segments of the lower limbs upon each other (dorsal flexion of the foct, flexion of the leg on the thigh, and the thigh en the pelvis). (2) Absence of the usual exaggeration of tendon reflexes, which mav be diminished, and in some cases even abolished. (3) A well-marked exaggeration of the "flexion reflexes" (Sherrington). ( 4 ) The almost constant presence of involuntary muscular contractions. There are also found objective sensory disturbances with a definite fixed upper limit, but the dominant clinical feature is the very obvious exaggeration of the flexion reflexes.

The condition indicates a severe and localized compression of the cord, and, in cases of tumour, is an indication for immediate operation.

Varying Clinical Picture at Different Levels.

At different levels of the cord certain additional symptoms may be added to the usual clinical picture, and may be of great localizing value. In high cervical tumours nystagmus is occasionally present, probably due to alterations' in the cerebro-spinal fluid pressure. Bulbar symptoms are occasionally seen, due, according to Schlesinger, to oedema of the medulla. Cranial nerve symptoms are very rare indeed. Intense pain may radiate up the back of the neck and head, giving rise to the stiffly held head suggestive of caries, or to an appearance of spasmodic torticollis.

The involvement of the roots of the phrenic nerve in tumour cases may result in paralysis of one or other side of the diaphragm, which can be verified by $x$-ray screening. C. P. Symonds has had recently under his care a most interesting case of tumour at the level of the third cervical segment, which commenced with persistent hiccup, lasting for a long time before the gradual onset of a quadriplegia. I have not been able to find a similar case reported in the literature, and therefore, with Dr. Symonds's permission, I will describe the symptoms very briefly.

The patient, a man aged 45, was admitted under Dr. Symonds's care in December, 1927, on account of spastic quadriplegia and attacks of hiccup.' Two years previously he had begun to suffer from attacks of hiccup lasting three or four days at a time, and so severe that they disabled him for the time being. Shortly after this he experienced pains in the back of the neck and across both shoulders. Six months later he began to notice weakness of his should limbs, and at the end of a further six months was a helpless
lower lis lower limbs, and at the end of a further six
invalid with weakness in all four extremities.

On admission the Wassermann reaction was negative, both in blood and cerebro-spinal fluid. The lumbar fluid showed a moderate excess of protein with no cells. Examination showed a spastic weakness of all four extremities, with a defect to temperature and pinprick extending up to the clavicles and including the upper limbs. The lower limbs showed increased tendon-jerss and extensor plantar responses. The abdominal reflexes were and extensor plantar "esponses. The abdominal reflexes were
absent. The refleses in the upper limbs were increased, and on scratching the skin on the anterior or posterior surface of the scratching the skin on the antericr or posterior surface of the
trunk at the level of either axilla a brisk reflex contraction of trunk at the level of either axilla a brisk reflex contraction of
the muscles of the appropriate shoulder girdle ensued, leading to retraction of the upper limb-the reflex described by Buzzard and Riddoch (Brain, 1021, 397) as occurring in spastic quadriplegia.

Whilst under observation the patient had a severe attack of hiccup lasting for three or four days and nights, and leading to great exhaustion.

In the lower cervical lesions four. root syndromes may occur-namely, (1) the ripper, or Duchenne-Erb type (C5, C6, C7); (2) the middie type (C7), with yaralysis and atrophy of the triceps; (3) the lower, or Aran-Duchenne type $(\mathrm{C} 8, \mathrm{D} 1)$; (4) the combination of the last with oculopupillary symptoms, Dejerine-Klumpke type (C8, D1, D2). The oculo-pupillary symptoms (Claude Bernard-Horner syndrome) are myosis, ptosis, enophthalmos, with narrowing of the palpebral fissure, together with vasomotor effects, as shown by redness or pallor of the skin, with hyperidrosis or anidrosis. It is in tumours of the cervical region that one sees the picture of a true spinal hemiplegia, which, however, soon passes into a quadriplegia. Paralysis of the upper limb may be of a spastic or atrophic nature, or may combine these characteristics. The radicular type of degenerative paralysis may be very clearly marked.

Tumours situated in the lower dorsal region of the cord may give rise primarily to pain simulating intra-abdominal disease, and every conceivable abdominal operation has been performed in such cases, in the erroneous belief that one cr other abdominal organ was at fault.

Tumours of the lumbar cord may show multiple root signs with atrophic paralysis, but in other cases pressure on the cord may cause the outstanding symptoms.

Tumours of the cauda equina give rise to intense pain, which may be widespread, in the small of the hack or over the sacrum, and which radiates down the back of the legs, into the region of the anus, bladder, perineum, and genitalia. These are cases which are usually treated for "sciatica" over long periods. Anaesthesia over the anogenital region, extending down over the tendo Achillis, with loss of the anal reflex and Achillis jerk, with bladder and rectal disturbances, make a very distinctive clinical picture. Trophic sores are peculiarly frequent in lumbosacral and cauda equina tumours, in marked contrast to those at higher levels of the cord.

A brief reference should be made to the so-called " atypical" clinical forms, or those cases which do not conform to the usual clinical picture produced by the presence of a spinal cord tumour. These are: (1) those with purely medallary symptoms; (2) those with purely root symptoms; (3) those with multiple tumours; (4) those with symptoms referred chiefly to the vertebral column and the paravertebral muscles-" forme rachialgique" or "pseudo-pottique" of French neurologists.

In the purely medullary form there is found a paraplegia without any signs of root involvement, either subjective or objective. According to Frazier and Ellis this form occurs once in every fourteen cases, and to Foerster in more than one-half of the cases. It is undoubtedly of frequent occurrence. It is explained upon the assumption that the tumour occupies an interradicular position, or lies on the anterior aspect of the cord, is of small size, and attached to the dural sheath. According to Oppenheim, it occurs with extradural tumours occupying an anterior position and of very soft consistence. 
In the purely root form the svmptoms are those of an ordinary neuralgia of varying distribution according to the site of the tumour. Attention should be directed to the obvious root limitation of the painful phenomena, to their persistence and long duration, and to the interference with the tendon or cutaneous reflexes at the corresponding level. In cases of neuralgia of long standing, with recessions, and which resist all forms of medication, lumbar puncture should be performed and the cerebro-spinal fluid examined, and if confirmatory evidence is necessary, followed by the injection of lipiodol.

The diagnosis of multiple tumours-diffuse sarconatosis, neurofibromata, diffuse gliomatosis-sometimes offers very great difficulties. They are to be suspected by the presence of two root syndromes clearly superimposed, which are accompanied by other symptoms of compression of the cord. There may be symptoms of a high compression with a root syndrome lower down, or the reverse, in which the root syndromes are very much higher than those of the cord compression. Clinically a diagnosis with localization of the highest tumour is usually made. Help may be obtained in these cases from the use of lipiodol.

The vertebral form is met with especially in tumours of root origin compressing the lower end of the cord or the roots of the cauda equina. It is marked by the intensity and diffuseness of the pain, which is referred to the pararertebral muscles and down the lower limbs. In an attempt to relieve the vertebral pain a secondary contracturelocalized or diffuse-of the affected muscles is induced. It is when it is localized that a diagnosis of Pott's disease is so often made. In the case of tumour the pain has an intermittent evolution, often with prolonged remissions, and the appearance of objective neurological signs is often slow. Again, the rigidity of the spine is not absolute, some slight movement of the spinous processes upon one another being obtained, and it is much more diffuse than that of Pott's disease. Moreover, the contracted muscles are excessively painful, and are tender to pressure.

\section{Differential Diagnosis of Tumours of the Spinal} Cond.

There is no symptom which occurs exclusively with any one form of spinal tumour. The individual symptoms are essentially the same, and differ in sequence, duration, and time of appearance according to the localization of the tumour.

As between extramedullary tumours, the intradural occur more frequently than the extradural in the proportion of three to one. The extradural tumours grow, as a rule, more quickly than the intradural, and hence, with them, the course of the symptoms is often more rapid, so that a short history is in favour of an extradural tumour rather than an intradural one.

Bony changes, as shown by the $x$ rays, are frequent in association with extradural tumours, while they are rarely seen with intradural, except in the so-called giant tumours of the cauda equina, which may cause an enlargement of the vertebral canal. Sensitiveness to pressure over the rertebral column is more frequent with extradural tumours. Root pain is more often absent with extradural tumours, and the spinal cord symptoms associated with them tend at first to be rague and indefinite.

It is especially with extradural tumours that lumbar puncture may produce a sudden and marked increase in the subjective and objective symptoms, though this is also true of intradural tumours attached to the dura. The crebro-spinal fluid does not show xanthochromia so often with extradural tumours, nor is its protein content ever so high. Greenfield says that a great excess of protein in the lumbar fluid, combined with a completely normal cisternal fluid, is in favour of an extradural rather than an intradural tumour. Moreover, with an osteoma or chondroma there may be no evidence of any subarachnoid block. According to Elsberg, the so-called "reversed", Brown-Séquard syndrome-contralateral motor and homolateral sensory disturbances-are often seen with extradural tumours.

It is often difficult chinically to differentiate an intraniedullaiy from an extramedullary tumour; the differences between the two are often misleading, and the diagnosis of an intramedullary tumour may be one of probability rather than of certainty. As a rule, in intramedullary tumours the root pains are less frequent, though they nay be present; the Brown-Séquard syndrome is rarer, and the paraplegia is less spastic. Moreover, amyotrophic phenomena are more frequent and may be of localizing value. A typical and clearly shown dissociated anaesthesia is greatly in favour of intramedullary tumour, although a more marked alteration in painful and thermal sensibility, as compared with other sensory disturbances, is not rare in extramedullary tumours.

In favour of a tumour being extramedullary rather than intramedullary, one may set forth the following points:

1. A preliminary stage of root pain, the signs of irritation preceding those of destruction.

2. The slow but continuous development of symptoms showing alterations in motor and sensory function.

3. The motor and sensory symptoms, while increasing in intensity, show a tendency to maintain a stationary localization. In other words, the growth of the tumour tends to be in breadth rather than in length.

4. The persistence of a Brown-Séquard syndrome for a fairly long time.

5. A marked degree of spasticity, which persists after the appearance of a complete paraplegia, together with early and pronounced development of the reflexes of defence in the whole of the lower extremity, with Babinski's sign well marked. 6. Symptoms of motor root irritation-cramps, spasms, tremor, muscular tension-and absence, or only slight development, of signs of atrophic degenerative paralysis.

7. Absence of trophic troubles in the soft tissues.

8. Changes in the cerebro-spinal fluid included in the term " loculation syndrome.",

\section{Aids to Diagrosis and a Lever Localization.}

These may be considered under the following headings: (1) Combined cisternal and lumbar puncture. (2) Evidence of a different chemical composition of the two cerebrospinal fluids (cisternal and lumbar). (3) Pneumomyelography. (4) Cisternal injection of lipiodol.

The method of combined cisternal and lumbar puncture has been elaborated by Arer as a means of determining the presence or absence of spinal subarachnoid block. Queckenstedt, in 1916, using lumbar puncture alone, showed that the pressure in the lumbar canal rose on compression of the jugular veins when there was no spinal block, but did not do so in the presence of an obstruction. This obstruction mist, however, be complete. Ayer's method is based upon the facts that in the normal individual the cisternal and lumbar fluids are practically identical: their pressures are the same, and oscillations due to coughing, sncezing, holding the breath, or to compression of the jugular veins are of similar range. Although diminished pressiure below the block is usual, normal pressures are not uncommonly found with tumours causing only a partial block, and occasionally an increased pressure may be found. This last may be due to a valvular action of a tumour, extramedullary in position, allowing a downward but preventing an upward flow of the fluid. Exudation of serum from the distended spinal veins may be also a contributory factor.

Normally the total amount of protein in the cerebrospinal fluid is approximately $25 \mathrm{mg}$. per $100 \mathrm{c.cm}$. of fluid. In cases of spinal block this figure may be increased a hundredfold in the stagnant fluid below the block. In addition, there also accumulates an excess of other substances, such as mineral salts and glucose, while the engorgement of the spinal vessels leading to a state of increased meningeal permeability causes the spinal fluid gradually to approximate in chemical composition to the blood plasma. Consequently in the fluid we find elements derived from the blood stream which are normally absent-altered blood, for example, producing the characteristic yellow coloration known as xanthochromia, whilst the presence of fibrinogen may cause the fluid to clot spontaneously in the test tube.

The outstanding features in the cerebro-spinal fluid below an area of spinal compression are therefore: (1) xanthochromia, (2) increase in the amount of protein, and (3) occasional spontaneous, coagulation. These three conditions are sometimes spoken of collectively as the "loculation syndrome." Occasionally a condition of spinal fluid stagnation is encountered in the fluid lying immediately abore a block, as shown by increased protein 
content, with or without xanthochromia. This is to ke explained by a transudation into the fluid from engorged veins in the spinal canal. As between demonstrable block and increased protein content, there is no doubt that the latter is a more delicate indication of cord compression or meningeal involvement.

The replacement of the cerebro-spinal fluid by air by means of lumbar puncture. was devised independently by Dandy, Bingel, and Wideröe. The procedure is briefly as follows. With the patient recumbent but at an angle of 50 to 60 degrees, $10 \mathrm{cccm}$. of cerebro-spinal fluid are withdrawr by lumbar puncture and an equal amount of air injected. In the absence of any spinal block the air passes at once into the cranial subarachnoid space, causing a fairly sharp pain, successively localized by the patient in different parts of the head. Aceording to Dandy, so constant and characteristic is this pain that it can be taken as positive evidence- of - the absence of a complete spinal block. To the pain are sometimes added nausea and voniting, a rise in temperature, and collapse. In the presence of a spinat block no such pain is complained of, and Dandy then advise, the injection of as much.air as the spinal canal will hold, in order to obtain the maximum effect of the ais-shadow for the $x$-ray localization of the site of the block. He finds a single stereosenpic plate, with the patient lying on the back and the rays passing straight through, gives the best result. The method is valueless in the cervical region when shadows, due to the tracheal air, obscure the picture.

The cisternal injection of lipiodol was introduced by Sicard and Forestier of Paris. Its technique is too well known to require description here. Passing over the symptoms which may arise as a result of the injection of the lipiodol, the question is how much reliance can be placed upon lipiodol localization. Can medullary compression be excluded with a negative lipiodol result? Assuredly not. There is no doubt that with small tumours, or in rare cases where narrowing of the cord results from destructive new growths, such negative results will occur. Again, cases occur in which the, nemological and lipiodol levels do not correspond. In other words, there is a sensory ipiodol dissociation. In some cases the sensory level is the higher, and in others the lipiodol. In the one case the sensory lipiodol dissociation may be explained on the assumption that the higher sensory level is due to the pressure of the stagnant fluid above the tumour and a localized ischaemia of the cord. In the other the lipiodol may be held up by meningeal adhesions above a tumour. or, in meningitis serosa circumscripta, at the level higher than the place where actual compression of the cord begins.

Given a sharply marked sensory level, I much prefer to depend upon it alone and to operate without the use of lipiodol. It is in cases in which the sensory level is not sharp and well defined, but gradually fades away as one passes upwards, that the lipiodol has its greatest field of usefulness.

May I quote here from my Lettsomian Lectures the rules which I formulated regarding the use of lipiodol in the diagnosis and localization of spinal compression.

"1. Its use should in no way usurp the place of careful and repeated systematic clinical examinations of the patient, especially with reference to a sensory level, combined with laboratory tests of the cerebro-spinal fluid. Recourse to it as a labour-saving device and a short cut to diagnosis and localization cannot be too strongly deprecated.

"2. It should only be used after a subarachnoid llock has been first demonstrated by the combined cistern-lumbar puncture method introduced by Ayer.

' 3 . It should not be used unless the possible dangers and complications are outweighed by the more exact localization likely to be obtained.

"4. Finally, in properly selected cases, we have in lipiodol a definite aid in the study of spinal cord compression, which, if used intelligently, will increase definitely our successful removal of spinal cord tumours at an earlier stage in the course of the disease, and thereby increase our percentage of cures and reduce our operative mortality."

\section{Treatment.}

There is only one treatment for spinal cord tumoursnamely, their removal by operation. This demands both - correct diagnosis and a precise level localization. These can only be arrived at by a careful history and by repeated systematic clinical examinations of the patient, especially with reference to a sensory level, combined with laboratory tests of the cerebro-spinal fluid. In doubtful cases may ba added such subsidiary aids as have been already alluded to.

The determination and locating of the spinous processes and their relation to the segments of the cord is allimportant. It is well known that the segments of the cord do not correspond to the similarly named vertebrae, and there is consideratile variation in the relation between the two. A serviceabte rule is that the lower cervical segments are each two vertebrae higher, the upper six dorsal three, and the lower six dorsal four, than the corresponding vertebrae. The lumbar segments correspond to the tenth and eleventh dorsal spines and the sacral to the twelfth dorsal and first lumbar. In oryler to locate a spinous process, commence counting from some easily recognized laudnark-for example, the seventh cervical or first dorsal spine-and count downwards to the desired spinous process, and then confirm this by counting upwards from the fourth lumbar spine, an easily recognized one. In very stout individuals it may be necessary to mark one spine with a small lead plate and then have the entire spine $x$-rayed in order to localize it accurately.

1 have no experience of usteoplastic laminectomy, nor of hemilateral or milateral laminectomy. Apart from the fact that osteoplastic operations are more difficult and take a longer tims, they all interfere with free access to the cord, and have no acivantages. They were devised on the erroneous supposition that removal of the spinous processes and laminae weakened the spine and interfered with its fice movement. The same objections hold good against a milateral laminectomy. The best type of operation is the one most rapidly done and which gives the best possible exposure of the tunour, thus enabing it to b. the more easily removed with the least danger of any damage to the cord.

In liny opinion, the operation of laminectomy as devised and developed by Victor Horsley; and which is still carried out at the National Hospital, Qmen Square, represents the simplest and best procedire for the exposure and removal of spinal cord tumcurs. The anaesthetic is ether given by the intratracheal method. If a general anaesthetic is contraindicated, local anaesthesia, either by Frazier's or by Heidenhain's method, may be used. The position of the patient may be prone, of semiprone, or lateral. I prefer the latter; it interferes less with respiration, opens out the arches of the laminae at the site of the operation, and allows of better drainage of fluids during the operation, thus doing away with the necessity of frequent sponging, which, however, may be obviated by the use of a suction apparatus.

The skin incision is made in the middle line directly over the spinous processes which are to be removed, and carried down at once to their tips, which are exposed. The fascia and muscular attachments are then freed from the spinous processes by cutting against the bone, and reflected on either side. With a broad raspatory the muscles and periosteum are cleared cleanly off the laninae on one side, deeper and deeper retraction being used as the procedure is carried out. Any bleeding is stopped by packing gauze into the cavity, and it is rarely necessary to use artery forceps. The same procecture is carrird ont on the other side. With the muscles well retracted, the spinous processes are removed by cutting through their bases with strong bone-cutting forceps. The laminae are then removed from below upwaras by cutting through on either side with bone forceps. A chisel-and hammer should never be used, and a saw is quite unnecessary. The epidural fat is now exposed, and when removed the dura is laid hare. Close inspection should now be made to see if the dura pulsates, or whether any extradural tumour is visible or is distorting the shape or position of the dural sac from pressure on its ventral surface. Any manipulations of the dural sac must be of the gentlest. A large size malleable probe may be passed very carefully upwards and downwards, first on the dorsal and then on the ventral surface of the dura, to see if there is any obstruction beyond the limits of the exposed area of dura. 
Havin reciled to open the dura, it is seized and steadied $b:$ a pair of fine-toothed forceps and a small opening is nade in a vertical direction with a fine knife. The openin: is then enlarged to the full extent of the exposed dura, the cut edges of the dura being seized and retracted with fine long artery forceps, or by traction sutures passe? with a fine round-bodied curved needle. To absorb any slight oozing of blood it is advisable to place small pieces of ganze ("cigarettes" or pieces of dental roll) on each side of the dural sac against the cut edges of the laminae. This keeps the operation field clear and prevents blood escaping into the thecal canal.

If the tumour lies in its most frequent situation-that is, on the postero-lateral aspect of the cord-it is at once visible. If it lies on the ventral aspect of the cord, the latter will be pushed backwards and to one side. Should no tumour be discovered, close inspection should be made as to the presence or absence of cardiac and respiratory pulsations in the cord, the condition of the posterior veins, and as to whether cerebro-spinal fluid continues to escape or not.

The presnuce of pulsation, the combined escape of cerebrospinal fluir, and a normal condition of the veins is strong evidence that the exposure is too high; the contrary conditions, that it is too low. Careful exploration on both aspects of the cord with a large knob-ended probe, bent at an apprepriate angle, in either an upward or downward direction as indicated, may give evidence of a definite block beyond the exposed area. Elsberg advises also the compression of the jugular veins (Queckenstedt's phenomenon) as being very useful in determining whether one is above or below the tumour level. If a free gush follows the compression it is presumptive evidence that one is above the tumour, and vice versa. In either case the laminectomy is extended in the direction indicated.

After remeral of the tumour the dura is closed by an interrupted or continuous suture of fine catgut. The gauze rclls on either side of the dural sac are removed, the muscles are united, layer by layer, with interrupted sutures of strong catgut, and the fascia covering them in like manner. The skin incision is closed with interrupted silkworm-gut sutures. If an extensive laminectomy has been performed in the cervical region, it is as well to incorporate a well-padded long light splint, extending from the middorsal region to beyond the head, in the dressing.

Technique for the Remoral of the Tumour.

The most important point in the removal of spinal cord tumours is the aroidance of any injury to the spinal cord. Their removal should always be attempted by working away from the cord. If possible, the cord itself should never be handled with fingers or instruments, and any sponging necessary must be of the very gentlest. Any nerve roots implicated should be gently disengaged. from the tumour, care being taken not to make traction on them away from the cord.

Should one or more posterior roots be incorporated in the tumour they should be divided. It is better to do this than to incise the tumour in an attempt to free the roots. If an anterior root is involved every endeavour should be made to preserve it, but occasionally this is impossible and it has to be divided. One should endeavour always to remove the tumour in one piece with its capsule.

If the tumour is meningeal in origin (the so-called dural endothelioma), and dorsal or dorso-lateral in situation, it will usually be lifted clear of the cord on retraction of the incised dura. It is very seldom adherent to the cord itself, though it may be to the posterior root or a slip of the dentate ligament. The dura is incised around the attachment of the tumour and remored with it. Before doing this it is well first to separate the tumour from any attachments it may have to cord, root, or ligament.

If the tumour lies on the rentral or ventro-lateral aspect of the cord its removal is one of greater difficulty. This may be lessened by a more extensive removal of the bone on the same side as the tumour. To displace the cord lying over the tumour a slip of the dentate ligament is seized in fine forceps and cut from its dural insertion. By traction on this the cord is rotated and drawn to the opposite side. Additional ease of approach, if required, can be obtained by also cutting one posterior root above and below the severed dentate ligament. It is better to do this than to risk damage to the cord by forcible displacement of it by instruments.

A tumour lying among the roots forming the cauda equina may give rise to great difficulty in its removal, owing to several, or even many, of the roots being incorporated in the tumour growth. In may therefore result in only so much tumour being removed as can be accomplished without doing irretrievable damage to a large number of roots; or, as advised by Elsberg, the operation may be done in two stages, the dura being opened and the tumour exposed at the first, and ten days to a fortnight later the wound reopened. It may then be found that the tumour has freed itself from many of the roots previously involved, and can be more safely removed, either in whole or in part.

\section{Removal of Intramedullary Tumours.}

This is best attempted by the "extrusion" method introduced by Elsberg. Its basic principle is a decompression operation with exposure of the tumour, followed by an interval of time to allow the increased pressure within the cord to extrude the tumour, in whole or in part. Having satisfied oneself that the tumour is intramedullary, the swelling is first aspirated with a fine hypodermic needle to exclude the possibility of its being due to fluid. If no fluid is obtained, an incision is made very carefully and slowly by means of a fine von Graefe knife into the cord, just to one side of the postero-median septum, to avoid the series of small median arterial branches which enter the posterior fissure. As soon as the tumour is exposed it will begin to extrude itself, but no attempt is made to remove it. A piece of Cargile membrane is placed over the cord and the wound closed, leaving the dura unsutured. A week later the wound is reopened, when it will be found that, if the tumour is localized, it will have been almost entirely extruded from the cord, and can be easily remored. If it is an infiltrating growth, the extruded portion can be removed and the remainder left undisturbed. Should no extrusion of the tumour take place, however, it is wiser to be content with the decompression only, rather than risk damaging the cord by attempting to remore the tumour. Good results have followed such a procedure at my hands.

Benign extradural growths, as a rule, offer no great difficulty in their removal, unless they extend all around the dura or lie on its rentral surface. It is a sine qua non in these cases that the bune be widely removed on one or both sides, as may be necessary, to obtain easier approach. Several posterior roots may have to be sacrificed in order to allow of displacement of the dural sac sufficiently to gain access to the tumour. In cases of osteoma or chondroma growing backwards from a vertebra or intervertebral disc, it is easier to open the dura, rotate and displace the cord as previously described for intradura! growths, and then cut through the dura lying over the tumour and remoie it through this opening.

The discussion of the treatment of spinal cord tumours would not be complete without the inevitable reference to $x$ rays and radium therapy.

Elsberg has never seen any permanent good results from $x$ rays or radium in irremovable intramedullary growths, but in some irremovable extradural tumours he says that the progress of the disease seemed to be checked for a time. Frazier says that it is incumbent upon the surgeon, in the presence of an inoperable growth, to resort to the use of one or other of these agencies, the selection to be left to the $x$-ray specialist. Peiper ${ }^{2}$ is of opinion that it is a mistake to attempt to replace operation by irradiation in the treatment of spinal tumours.

French authorities are more enthusiastic, though cautious. Sicard, Gally, Haguenan, and Wallich consider that radiotherapy is efficacious in all types of tumour, but only in a small number of them. As a sequel to operation they advise its use only in gliomata and endotheliomata, four or five weeks subsequently, if the symptoms persist, but not in benign growths. They attach great importance to the cerebro-spinal fluid below the lesion. If it is normal, in a case in which the paraplegia has become 
stationary after post-operative improvement, radiotherapy is useless. They end up with a warning against exaggerating the definite curative value of radiotherapy, and call upon the pathologists to adopt a universal terminology for the different types of tumours, and at the same time to group then according to their radio-sensibility or their radio-resistance. IJourn. Amer. Med. Assoc., REFERENCES.
October 24th, 1927. 2 Deut. med. Woch.,

\section{THE CONTROL OF DIPHTHERIA AND SCARLET FEVER.}

\section{BY}

R. A. O'BRIEN, C.B.E., M.D.,

Wellcome Physiological Research Laboratories, Beckenham, Kent.

\section{DIPHTHERIA}

I ANTICIPATE that those who join with me in opening the discussion will agree that with swabbing, testing for virulence to detect the true diphtheria bacillus, Schick-testing, active immunization with prophylactic, and, in emergencies, passive immunization with serum, we can control an outbreak or prevent outbreaks-if the population concerned is disposed to take full advantage of these measures. Just as surely as Pasteur and his hostile critics, when they saw in the dawn on the farm at Pouilly-le-Fort the vaccinated sheep quite well while the unvaccinated lay dying of anthrax, knew that complete control of that disease was available to those who wished, so the medical administrators of the large fever hospitals and residential schools know that they can promise the almost complete abolition of diphtheria amongst the nurses or pupils. Applied to the general population the problem passes out of the narrow field of experimental observation on small groups of people with complete controls, and other considerations, economic, social, and political, play a part.

The Practitioner's Part in Control.

The control of any. infectious disease rests upon the joint work of the general practitioner and the public health authorities. From communications with general practitioners I would with considerable trepidation suggest that perhaps the busy practitioner, faced with the call for quick decision in daily practice, would welcome a short didactic statement by the Association somewhat on the following lines:

Patient.-If a patient has the smallest amount of membrane in the throat or nose, or any sudden difficulty in breathing, and any suspicion of diphtheria arises, take a swab if you wish, but gire antitoxin at once. The risk to life from the act of giving serum is probably 1 in 60,000 , whereas the average risk to life from diphtheria is 3 to 10 per cent.; every hour's delay in giving serum increases the risk.

Contacts.-The ideal course is to test all contacts by Schick's method and, after separating the Schick-negative reactors-amongst whom will be found any dangerous "carriers" of virulent bacilli-concentrate attention on those giving a positive reaction. See them daily (in order to detect cases of diphtheria at the earliest moment and give serum immediately), or, if that is not possible, consider protecting this group with serum. After the emergency is over, protect them by actire immunization. If the Schick test cannot be done the general line of action is the same-that is, inspect daily, or, if necessary, protect with antitoxin.

\section{Responsibility for Deaths from Diphtheria.}

In the IVeekly Bulletin of the City of New York Department of Health for February 5th, 1927, occurs the following statement:

"In diphtheria there were 56 such deaths. The parent delayed in calling a doctor from, one to five days in 46 cases, usually because 'home remedies' were considered sufficient. The doctor delayed the administration of antitoxin for a period of from one to fourteen days in 29 out of the 56 cases. In 14 cases the

* Read in a discussion in the Section of Preventive Medicine of the Annual Meeting of the British Medical Association, Cardiff, 1928. doctor's first diagnosis was wrong and in 13 edses he was doubtful. The wrong diagnoses were usually colds and tonsilitis, pneumonia, indigestion, and in one case tecthing."

In 1920 Carey $^{1}$ published a valuable analysis of 1,000 deaths from diphtheria in the State of Massachiusetts. He points out the difficulty of obtaining positive cultures in the early stages of laryngeal diphtheria and also that the nasal type had been frequently "missed."

With regard to the parents' responsibility, 23 per cent. of the children were ill a week, and 4 per cent. ill from one to two weeks, before a doctor was called in.

“An alarming percentage of 11.8 of our cases were found moribund upon visitation by the physician. . . . In some instances it was noted that physicians waited for a laboratory repori from their cultures before administering antitoxin. ... In 29 in-lances it was found that less than 3,000 units of antitoxin were administered. - The usual doses, however, seem to have been from 6,000 to 9,000 units $\ldots$ in no instance did we find antitoxin given intravenously.... It is most earnestly recommended that this procedure be used in those cases which are seen late in the disease.

I have been unable to find any English official figures of the kind, but probably a medical officer of health with long experience in any large English city could produce somewhat similar records. A confidential " inquest" into every death from diphtheria by a tactful medical officer of health who has the complete confidence of the practitioners in his area would probably yield information of value. The ascertainment of the "parents' responsibility" for late summoning of the doctor would give data of use for public propaganda, and any information relating to delayed medical diagnosis or too small dosage with antitoxin would obviously be of value to the medical men concerned.

\section{Gaps in our Knowledge.}

There do not seem to be readily available any large groups of figures indicating how long the Schick-negative condition lasts. Parish and Okell have recently completed a valuable survey which will shortly be published. I am indebted to them for permission to quote some of the figures. In approximately 1,000 children re-tested from one to seven years after giving a negative response, 97 per cent. gave a completely negative Schick reaction. Any reaction not clearly negative was read as "positive." About 500 of the children had originally been " naturally" negative to the Schick reaction; of these 99 per cent. remained negative; 4 of those read as "positive" were potentially immune, and rapidly developed antitoxin as the result of the Schick re-test; only 2 were definitely positive with no circulating antitoxin and required reimmunization. Amongst about 450 who had been originally immunized until they showed a negative reaction, $95 \mathrm{per}$ cent. remained negative, 5 per cent. were positive or " faintly positive," and became negative as the result of the first re-test, while again 2 "immunologically obstinate" children, brother and sister, required re-immunization.

\section{Degree of Immunity Represented by a Schick-negative} condition.

Another gap concerns the degree of immunity represented by a Schick-negative or " immuns" condition. "Immunity" is relative and not absolute. From the point of view of immunity against diphtheria the population of an ordinary town cannot be divided into sharply defined groups of "white" (or " immune") and " black" (or " non-immune")-there are a few of varying shades of grey. In large groups immunized long ago there are somewhat more in the "grey" section.

When the diphtheria bacillus attempts to attack the "white immune" its attack fails; it prevails against the non-immune, and clinical diphtheria occurs. In the case of the "grey," whether the attack succeeds or not depends on the weight of the attack and on the amount of white (or grade of immunity) in the grey-that is, whether the attacked has available a store of antitoxin in his blood, and, probably more important, whether his cells aro trained and alert so that they can produce speedily and abundantly the needed antitoxin. Fortunately this potential immunity remains even when the circulating antitoxin has, long after a successful course of immunization leading to the production of a Schick-negative condition, fallen to a very small amount-that is, less than one two-thousandth of a unit of antitoxin per cubic centimetre. The indi- 